\title{
AVALIAÇÃO DA SOLUBILIZAÇÃO DE PARAFINAS POR MICROEMULSÃO UNITOL L90/ N-BUTANOL/ ÁGUA/ QUEROSENE
}

\author{
Y. K.P. G. AUM ${ }^{1,2}$, M. C. C. de $\mathrm{SA}^{1}$, P. T. P. AUM ${ }^{3}$, E. L. BARROS NETO ${ }^{1}$ e T. N. de CASTRO \\ DANTAS $^{4}$ \\ ${ }^{1}$ Universidade Federal de Rio Grande do Norte, Departamento de Engenharia Química \\ ${ }^{2}$ Universidade Federal do Amazonas, Departamento de Engenharia Química \\ ${ }^{3}$ Universidade Federal do Rio Grande do Norte, Programa de Pós-Graduação em Ciência e \\ Engenharia de Petróleo \\ ${ }^{4}$ Universidade Federal do Rio Grande do Norte, Departamento de Química \\ E-mail para contato: yanne.aum@gmail.com/yanne@ufam.edu.br
}

\begin{abstract}
RESUMO - As operações de produção de petróleo offshore estão cada vez mais desafiadoras, submetendo o petróleo às grandes variações de temperatura e pressão, desde o reservatório até a superfície, e culminando muitas vezes na precipitação de cristais de parafinas. Esses cristais acabam se acumulando na superfície das tubulações, reduzindo o fluxo de produção e, chegando muitas vezes, ao ponto de plugá-las e interromper a produção. Portanto, visando propor uma alternativa para lidar com esse problema, este trabalho estudou o uso de sistemas microemulsionados formados por Unitol L90/n-Butanol/Querosene/Água na solubilização de parafinas e a influência da temperatura na capacidade de solubilização. Os resultados mostraram maior capacidade de solubilização pela microemulsão do que pelo solvente orgânico, o querosene, em uma faixa maior de temperaturas. Esses resultados indicam a possibilidade de aplicação das microemulsões avaliadas na remoção de depósitos parafínicos em tubulações.
\end{abstract}

\section{INTRODUÇÃO}

O processo de deposição de parafinas é um dos principais problemas do transporte de petróleo em oleodutos. Nesse processo, as parafinas presentes no petróleo, ao atingirem a temperatura de início de aparecimentos dos cristais, começam a se depositar nas paredes da tubulação mais fria (Huang et al., 2011). A deposição de parafinas restringe o fluxo de petróleo, promovendo o aumento na perda de carga no oleoduto, o que representa uma elevação dos custos operacionais e de reparação. Nos casos mais críticos, a contínua deposição de parafinas pode aumentar a espessura do depósito ao ponto de plugar a tubulação, causando a parada da produção (Akbarzadeh e Zougari, 2008).

Al-Yaari (2011) explanou sobre diversas técnicas empregadas pela indústria do petróleo na remoção de depósitos parafínicos, incluindo reações químicas exotérmicas, remoção mecânica, aquecimento, solventes, dispersantes, campo magnético, e tratamento biológico. Entretanto, todas as técnicas atualmente utilizadas apresentam problemas e limitações na sua aplicação. Pesquisas continuam sendo realizadas com o objetivo de encontrar métodos mais eficientes, seguros e com o 
melhor custo/benefício.

Nesse cenário, os sistemas microemulsionados, devido às suas características de alta estabilidade, capacidade de diminuir a tensão interfacial, capacidade de estabilizar dois componentes imiscíveis e elevada área interfacial formada entre a fase dispersa e a contínua; se mostram com grande potencial de aplicação na solubilização de depósitos parafínicos, visando a remoção da parafina depositada no interior de tubulações.

As microemulsões podem ser definidas como sistemas termodinamicamente estáveis, isotrópicos, transparentes, de dois líquidos imiscíveis, estabilizados pela presença de tensoativos (Robb, 1982). Portanto, visando avaliar a estabilidade do sistema microemulsionado Unitol L90/nButanol/Água/Querosene e sua capacidade de solubilização de parafinas em diferentes temperaturas, este trabalho foi desenvolvido e os resultados comparados a atuação do solvente querosene, com o objetivo de estudar a viabilidade do uso desse sistema como fonte alternativa de remoção de depósitos de parafinas.

\section{MATERIAIS E MÉTODOS}

Os materiais utilizados e os métodos adotados para a realização dos experimentos são apresentados neste tópico. A sequência das etapas do desenvolvimento deste trabalho é mostrada no fluxograma da Figura 1.

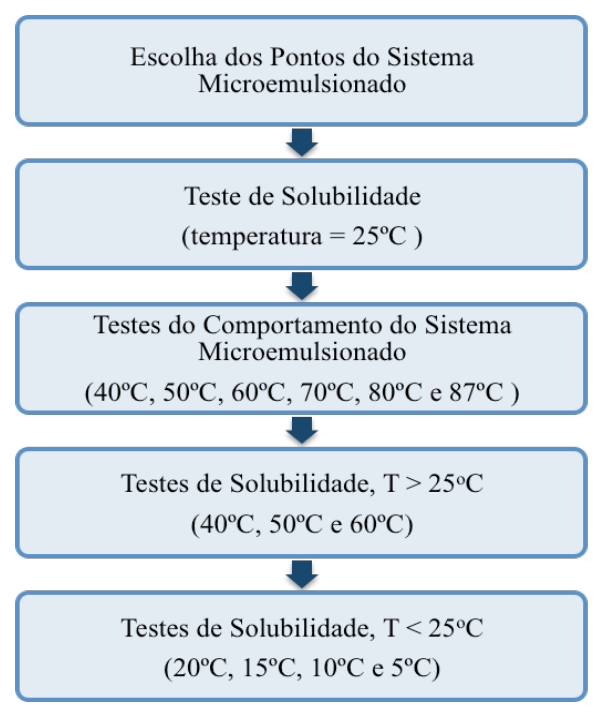

Figura 1 - Fluxograma das etapas de realização dos experimentos deste trabalho.

\subsection{Escolha dos Pontos de Microemulsão}

Com base no trabalho de Castro Dantas et al. (2010), no qual foram avaliados diversos sistemas microemulsionados na solubilização de frações pesadas de petróleo presentes em amostras de rochas de reservatório de petróleo, foi escolhido um sistema constituído de tensoativo/cotensoativo/fase aquosa/fase óleo estudado por esses pesquisadores e que apresentou uma maior região de microemulsão para ser avaliado neste trabalho. $\mathrm{O}$ intuito foi encontrar a maior faixa de concentração dos constituintes formadora de microemulsão para se trabalhar com diversas composições, assim o sistema Unitol L90 (tensoativo) + n-butanol (cotensoativo) + água 
(fase aquosa) + querosene (fase óleo) foi o escolhido para ser empregado no estudo de solubilização de parafinas neste trabalho. O diagrama de fases desse sistema é apresentado na Figura 2.

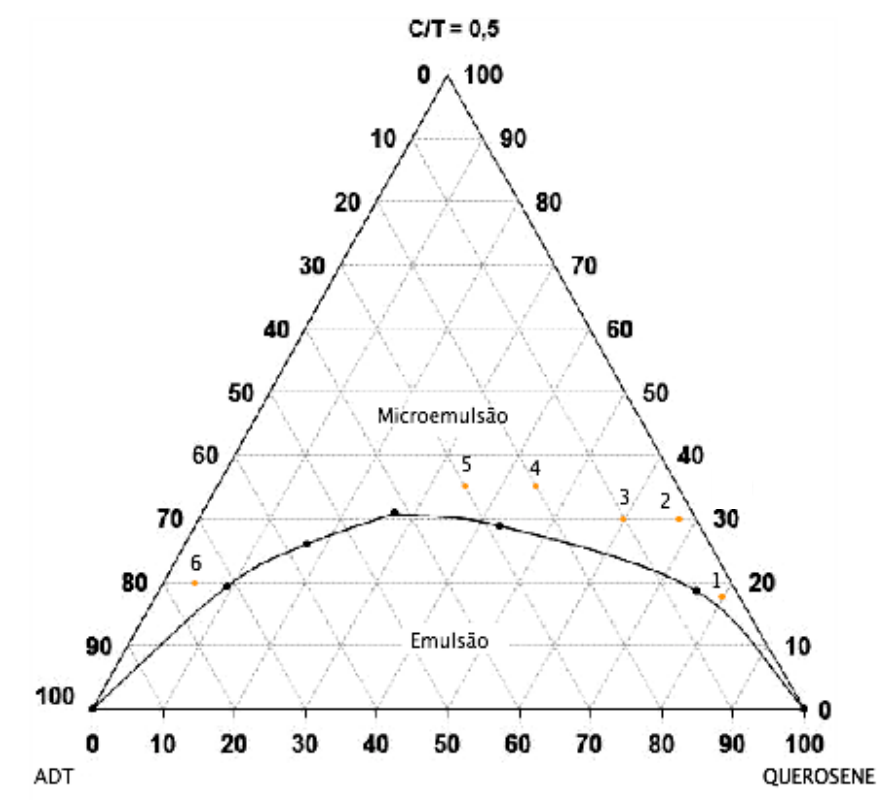

Figura 2 - Diagrama de fases pseudo-ternário do sistema: Unitol L 90 (T) + n-butanol (C) + água de torneira (ADT) + querosene, em temperatura ambiente $\left(25 \pm 1^{\circ} \mathrm{C}\right)$, adaptado. Fonte: Castro

Dantas et al. (2010). Pontos escolhidos para o estudo de solubilidade: 1, 2, 3, 4, 5 e 6.

Uma vez determinado o sistema a ser avaliado, foram escolhidos seis pontos no diagrama pseudo-ternário da Figura 2, localizados em faixas de interesse para o presente estudo, a fim de traçar um perfil de comportamento desse sistema como solubilizante de parafina, variando sua temperatura e sua composição. As composições escolhidas estão apresentadas na Tabela 1.

Tabela 1 - Composição mássica dos pontos de microemulsão escolhidos

\begin{tabular}{|c|c|c|c|c|c|c|c|}
\hline Componente & Ponto 1 & Ponto 2 & Ponto 3 & Ponto 4 & Ponto 5 & Ponto 6 \\
\hline \multicolumn{7}{|c|}{ Composição mássica percentual mássico no diagrama } \\
\hline C/T & $17,5 \%$ & $30 \%$ & $30 \%$ & $35 \%$ & $35 \%$ & $20 \%$ \\
\hline Água & $2,5 \%$ & $2,5 \%$ & $10 \%$ & $20 \%$ & $30 \%$ & $75 \%$ \\
\hline Querosene & $80 \%$ & $67,5 \%$ & $60 \%$ & $45 \%$ & $35 \%$ & $5 \%$ \\
\hline \multicolumn{7}{|c|}{ Composição correspondente em gramas } \\
\hline Unitol L90 & $1,16 \mathrm{~g}$ & $2 \mathrm{~g}$ & $2 \mathrm{~g}$ & $2,33 \mathrm{~g}$ & $2,33 \mathrm{~g}$ & $1,33 \mathrm{~g}$ \\
\hline n-butanol & $0,58 \mathrm{~g}$ & $1 \mathrm{~g}$ & $1 \mathrm{~g}$ & $1,17 \mathrm{~g}$ & $1,17 \mathrm{~g}$ & $0,665 \mathrm{~g}$ \\
\hline Água & $0,26 \mathrm{~g}$ & $0,25 \mathrm{~g}$ & $1 \mathrm{~g}$ & $2 \mathrm{~g}$ & $3 \mathrm{~g}$ & $7,5 \mathrm{~g}$ \\
\hline Querosene & $8 \mathrm{~g}$ & $6,75 \mathrm{~g}$ & $6 \mathrm{~g}$ & $4,5 \mathrm{~g}$ & $3,5 \mathrm{~g}$ & $0,5 \mathrm{~g}$ \\
\hline
\end{tabular}

\subsection{Avaliação da Solubilidade de Parafina em Microemulsão}

Um vez escolhidos os pontos de microemulsão do diagrama pseudo-ternário, Figura 2, nas faixas rica em óleo, rica em água e na região intermediária para os ensaios, primeiramente fez-se 
um estudo do comportamento destes como solubilizante de parafina na temperatura ambiente, sob agitação constante. A parafina utilizada foi a RLAM 140/145 ${ }^{\circledR}$, produzida, entabletada/pulverizada e embalada na Refinaria RLAM - BA.

Inicialmente, para reproduzir os pontos escolhidos, foi pesado (para cada ponto) em balança de alta precisão $10 \mathrm{~g}$ de microemulsão e acondicionado em um béquer. Em seguida adicionou-se $0,01 \mathrm{~g}$ de parafina em cada sistema, levando-o para agitação, em um tempo limite de $1 \mathrm{~h}$, antes do qual, se fosse observada completa solubilização já poderia adicionar outra quantidade de $0,01 \mathrm{~g}$ de parafina, e isso se repetia até que se atingisse a saturação, ou seja, até que o sistema fícasse turvo. Tal passo foi realizado à temperatura ambiente. $\mathrm{O}$ mesmo teste de solubilidade à temperatura ambiente foi feito para o querosene puro, utilizando, desta vez, $10 \mathrm{~g}$ de querosene.

\subsection{Avaliação do Comportamento dos Sistemas Microemulsionados Saturados com Parafina Submetidos ao Aumento de Temperatura}

Tendo sido alcançada a saturação da parafina de cada ponto de microemulsão, na temperatura ambiente, os sistemas foram submetidos a um banho termostático, às temperaturas de: $40^{\circ} \mathrm{C}, 50^{\circ} \mathrm{C}, 60^{\circ} \mathrm{C}, 70^{\circ} \mathrm{C}$ e $87^{\circ} \mathrm{C}$, a fim de analisar a influência da temperatura na solubilidade e, assim, selecionar o ponto que apresentou melhor comportamento, ou seja, aquele se manteve homogêneo, mesmo com a variação de temperatura, para servir como sistema de partida para os demais estudos.

\subsection{Avaliação da Solubilidade de Parafina em Microemulsão em Função da Temperatura}

Teste de solubilidade em temperaturas $>25^{\circ} \mathrm{C}$ : a solubilidade da parafina nos seis pontos de microemulsão escolhidos e no querosene puro foram determinadas, nas temperaturas de $40^{\circ} \mathrm{C}$, $50^{\circ} \mathrm{C}$ e $60^{\circ} \mathrm{C}$. Isso foi realizado adicionando a parafina aos sistemas de microemulsão e querosene, os quais se encontravam sob agitação e temperatura constantes, no Banho Maria Dubnoff com Agitação Angular TECNAL ${ }^{\circledR}$ TE-0532, até que eles se tornassem turvos. No banho, os béqueres são fixados por garras metálicas. Em seguida foi pesada a massa final dos sistemas e, sabendo a massa inicial antes de ter sido adicionada a parafina, por diferença é possível determinar quanto de parafina, aproximadamente, foi solubilizada, ou seja, qual a solubilidade naquela temperatura.

Teste de solubilidade em temperaturas $<25^{\circ} \mathrm{C}$ : neste experimento, os sistemas de microemulsão e querosene puro foram submetidos às temperaturas de $20^{\circ} \mathrm{C}, 15^{\circ} \mathrm{C}, 10^{\circ} \mathrm{C}$ e $5^{\circ} \mathrm{C}$ por um banho de resfriamento. As amostras foram alocadas em células de $50 \mathrm{~mL}$, acopladas ao Banho Termostático Julabo ${ }^{\circledR}$ Modelo F33 - MC, como mostrado na Figura 3. A massa das amostras foi adquirida da mesma maneira que no experimento de aquecimento, exceto da parafina que, nesse caso, foi adicionada a cada $0,01 \mathrm{~g}$ e assim pôde ser somada. 


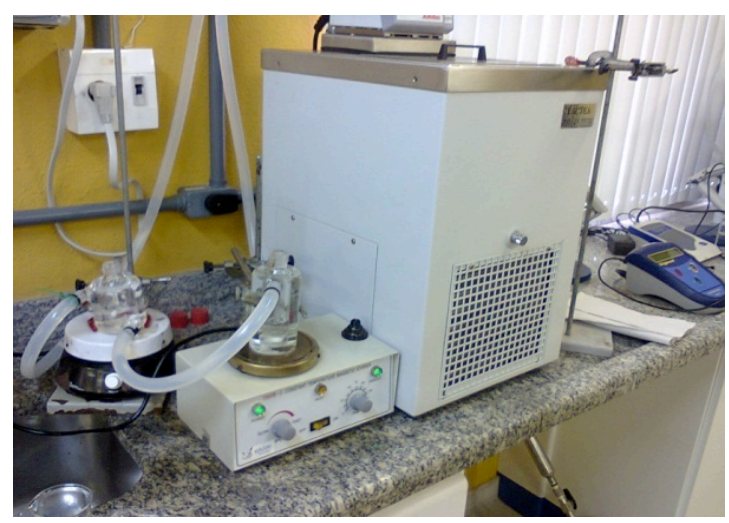

Figura 3 - Aparato experimental para realização dos testes de solubilidade de parafina nas temperaturas $20,15,10$ e $5^{\circ} \mathrm{C}$.

\section{RESULTADOS E DISCUSSÃO}

\subsection{Avaliação da solubilidade de parafina em microemulsão na temperatura ambiente}

A Tabela 2 apresenta a massa de parafina RLAM 140/145 adicionada que saturou o sistema, nas composições dos pontos de 1 a 6 , por $10 \mathrm{~g}$ de microemulsão ou $10 \mathrm{~g}$ de querosene.

Tabela 2 - Carga máxima de parafina nos pontos de microemulsão escolhidos em temperatura ambiente $\left(25 \pm 1^{\circ} \mathrm{C}\right)$

\begin{tabular}{|c|c|c|c|c|c|c|}
\hline Ponto 1 & Ponto 2 & Ponto 3 & Ponto 4 & Ponto 5 & Ponto 6 & Querosene \\
\hline \multicolumn{7}{|c|}{ gramas de parafina/ 10 gramas de solvente } \\
\hline 0,4263 & 0,4245 & 0,3300 & - & - & 0,0167 & 0,5569 \\
\hline
\end{tabular}

O ponto 1 pertence à região rica em óleo do diagrama pseudo-ternário. Logo, como era de se esperar, pelo fato da parafina ser facilmente solúvel em querosene, apresentou uma boa solubilidade da parafina na temperatura ambiente.

O segundo ponto foi escolhido também na região rica óleo, mas mantendo a mesma quantidade de água e aumentando a concentração de C/T para se observar como seria seu comportamento. Na temperatura ambiente, o sistema saturou (ficou turvo), à massa de $0,4245 \mathrm{~g}$ de parafina adicionada; apresentando um comportamento semelhante ao do Ponto 1; o que era de se esperar, já que ambos são ricos em óleo. Porém, vale ressaltar que o Ponto 1 é mais vantajoso para uso em grande escala do que o Ponto 2 no que diz respeito a gastos; tendo em vista que ambos apresentaram solubilidades próximas, mas o Ponto 1 utilizou menos tensoativo, que é um produto químico com valor comercial considerável.

$\mathrm{O}$ ponto 3 escolhido ainda pertence à região rica em óleo, mas agora foi mantida a concentração de $\mathrm{C} / \mathrm{T}$ e aumentada a concentração de água para observar como o sistema se comportava. Na temperatura ambiente, o sistema saturou (ficou turvo) à massa de $0,3300 \mathrm{~g}$ adicionada. Essa diminuição na solubilidade comparada aos Pontos 1 e 2 era esperada, uma vez que a parafina é insolúvel em água. 
O ponto 4 foi escolhido na região intermediária do diagrama, onde as proporções de óleo, água e razão $\mathrm{C} / \mathrm{T}$ são próximas entre si, a fim de avaliar a influência da temperatura na solubilidade de parafina com essas proporções nos próximos estudos. Quando preparado (na temperatura ambiente), o sistema se apresentou turvo, ou seja, como se não fosse pertencente à região de microemulsão. Talvez, o que tenha ocasionado tal problema tenha sido a proximidade do ponto à curva que delimita as regiões, o que, por alguns décimos de massa adicionada além do esperado, o tirou da região de microemulsão. Contudo, a fim de avaliar se era mesmo esse o problema ou se acontecia porque o sistema era instável com a temperatura, ainda foi adicionada parafina $(0,0135 \mathrm{~g})$ para analisar a sua solubilidade com a temperatura. $\mathrm{O}$ mesmo comportamento do Ponto 4 foi observado no ponto 5. Isso já era esperado, haja vista que pertencem a uma região do ternário com composições bem próximas e desfavoráveis para a solubilidade de parafina.

O ponto 6 é um representante da região rica em água, $75 \%(\mathrm{~m} / \mathrm{m})$, do diagrama. Como era de se esperar, já que a parafina é insolúvel em água, na temperatura ambiente o sistema se manteve transparente (uma vez que é uma microemulsão), mas com cristais da parafina adicionada $(0,0167 \mathrm{~g})$ em suspensão, mesmo depois de ser agitado por $1 \mathrm{~h}$.

\subsection{Avaliação do comportamento dos sistemas microemulsionados saturados com parafina submetidos ao aumento de temperatura}

Os resultados da avaliação do comportamento dos sistemas microemulsão-parafina saturados quando submetidos à elevação da temperatura apresentaram diferenças entre os sistemas. O sistema microemulsionado do ponto 1 com parafina, que ora estava turvo na temperatura ambiente, quando submetido ao aquecimento ficou límpido nas temperaturas de $40^{\circ} \mathrm{C}$, $50^{\circ} \mathrm{C}, 60^{\circ} \mathrm{C}, 70^{\circ} \mathrm{C}, 80^{\circ} \mathrm{C}$ e $87^{\circ} \mathrm{C}$; ou seja, manteve solúvel a parafina. Da mesma maneira, quando submetido ao aquecimento, o ponto 2 ficou límpido. Assim, o comportamento

$\mathrm{O}$ ponto 3 que se mantinha turvo à temperatura ambiente, ficou límpido no decorrer do aquecimento até a temperatura de $80^{\circ} \mathrm{C}$, quando turvou e se manteve assim na última temperatura medida, a $87^{\circ} \mathrm{C}$. Tal fato se deve à instabilidade da microemulsão nessa proporção diante de temperaturas elevadas. Por esse motivo este ponto foi descartado do próximo estudo. Nos pontos 4 e 5 foi possível observar que, durante o aquecimento, os sistemas se mostraram límpidos em $40^{\circ} \mathrm{C}$, e em temperaturas maiores ficaram turvos. Logo, notou-se que este ponto foi instável com a temperatura, sendo, então, descartado do interesse em novos estudos.

O sistema do ponto 6 permaneceu com uma fase límpida e um cristal presente até a temperatura de $70^{\circ} \mathrm{C}$, quando turvou. Porém, com um pouco de agitação, o sistema voltou ao estado inicial. O que se pode concluir desse comportamento é que, nesta proporção, rica em água, a microemulsão não solubiliza parafina. Portanto, também este ponto foi descartado.

Por fim, descartados os Pontos 3, 4, 5 e 6, nos restou os Pontos 1 e 2, dos quais um foi escolhido para os demais estudos. O critério para escolha foi, então (já que ambos pertenciam a uma mesma região rica em óleo), em qual deles seria necessário utilizar menos tensoativo, que é um produto químico relativamente caro em comparação ao querosene. Portanto, como no primeiro ponto a razão $\mathrm{C} / \mathrm{T}$ correspondia a $17,5 \%$ em massa da microemulsão, ao passo que no segundo ponto representava $30 \%$, o Ponto 1 foi escolhido. 
homogêneo mesmo com o aumento de temperatura, foi o sistema base de microemulsão sobre o qual foi estudada a influência da temperatura na sua capacidade de solubilização de parafina. $\mathrm{O}$ mesmo foi feito também, para efeito comparativo, com o sistema de querosene puro.

\subsection{Avaliação da solubilidade de parafina em microemulsão em função da variação de temperatura}

Teste de solubilidade em temperaturas $\mathrm{T}>25^{\circ} \mathrm{C}$ : a Tabela 3 apresenta os resultados encontrados para a microemulsão do Ponto 1 nas temperaturas de 40,50 e $60^{\circ} \mathrm{C}$.

Tabela 3 - Carga máxima de parafina em microemulsão (Ponto 1) e querosene para $\mathrm{T}>25^{\circ} \mathrm{C}$

\begin{tabular}{|c|c|c|}
\hline & Microemulsão (Ponto 1) & Querosene \\
\hline Temperatura & \multicolumn{2}{|c|}{ gramas de parafina/10 gramas de solvente } \\
\hline $25^{\circ} \mathrm{C}$ & 0,4263 & 0,5569 \\
\hline $40^{\circ} \mathrm{C}$ & 1,1111 & - \\
\hline $50^{\circ} \mathrm{C}$ & 7,0797 & - \\
\hline $60^{\circ} \mathrm{C}$ & 30,0233 & - \\
\hline
\end{tabular}

O aquecimento aumenta a hidrofilia do emulsificante, que irá absorver mais água do que óleo na fase microemulsão, ocasionando aumento de volume da fase óleo. Com esse aumento do óleo, devido ao fato dele ser a fase solubilizante de parafina, pode-se dizer que o aumento da temperatura acarreta na maior solubilização. Logo, partindo desse pressuposto, a quantidade de parafina adicionada em temperaturas maiores do que a ambiente deve ser maior do que nessas condições, como observado nos resultados da Tabela 3.

Houve uma diminuição na massa do sistema para os ensaios com querosene quando este alcançou as temperaturas de $40^{\circ} \mathrm{C}$ e $50^{\circ} \mathrm{C}$. Isso ocorreu porque o ponto de fulgor do querosene é de $40^{\circ} \mathrm{C}$. O ponto a $60^{\circ} \mathrm{C}$ foge desse comportamento porque a partir daí a parafina utilizada RLAM $140 / 145$, que tem ponto de fusão aproximadamente de $61,65^{\circ} \mathrm{C}$, uma vez no estado líquido, tornase um importante solubilizante da parafina sólida.

Teste de solubilidade em temperaturas $<25^{\circ} \mathrm{C}$ : os valores pesados para a reprodução da microemulsão utilizada para esse experimento e a massa de parafina nesse sistema estão listados na Tabela 4.

Tabela 4 - Carga máxima de parafina em microemulsão (Ponto 1) e querosene para $\mathrm{T}<25^{\circ} \mathrm{C}$

\begin{tabular}{|c|c|c|}
\hline & Microemulsão (Ponto 1) & Querosene \\
\hline Temperatura & gramas de parafina/10 gramas de solvente \\
\hline $25^{\circ} \mathrm{C}$ & 0,4263 & 0,5569 \\
\hline $20^{\circ} \mathrm{C}$ & 0,051 & 0,044 \\
\hline $15^{\circ} \mathrm{C}$ & 0,0232 & 0,0228 \\
\hline $10^{\circ} \mathrm{C}$ & 0,0117 & 0,01162 \\
\hline $5^{\circ} \mathrm{C}$ & 0,00623 & 0,00598 \\
\hline
\end{tabular}

É possível observar que a solubilidade diminuiu com a temperatura; o que era de se esperar, 
já que acontece o inverso do aumento da temperatura, quando o volume de óleo é expandido, consequentemente, por afinidade, mais parafina é solubilizada. Também para o querosene a queda de temperatura desfavoreceu a solubilidade da parafina.

A partir da Tabela 4, é possível notar que a microemulsão apresentou maior solubilidade de parafina nas temperaturas menores, em relação ao querosene. Mesmo sendo a diferença entre eles não tão expressiva, ainda são valores consideráveis, uma vez que estamos tratando de medidas de massa na ordem de dezena de miligrama. Assim como a solubilidade diminui com a temperatura para ambos, também a diferença entre as suas solubilidades diminui, o que nos leva a crer que a taxa de queda na solubilidade é maior na microemulsão do que no querosene puro.

\section{CONCLUSÃO}

Através da realização deste trabalho foi possível concluir que, embora o querosene tenha solubilizado mais parafina do que a microemulsão na temperatura ambiente, esta última se apresentou como sendo mais eficiente como solubilizante. Isto porque a microemulsão teve seu poder solubilizante aumentado com a temperatura, ao passo que o querosene foi vaporizado com o aquecimento. $\mathrm{O}$ que mostra que essa técnica pode ser empregada em conjunto com um tratamento térmico. Também nas temperaturas de $20,15,10$ e $5^{\circ} \mathrm{C}$ a microemulsão apresentou maiores vantagens quanto ao seu uso como solubilizante de parafina do que o querosene, pois, apesar de ter tido uma taxa de queda na sua solubilidade maior do que a do óleo puro, ainda apresentou maior capacidade de solubilização. Este aspecto é especialmente importante, pois atinge um ponto crucial da deposição de parafina nas tubulações de produção de petróleo, principalmente offshore: Isso nos leva a crer que, com o desenvolvimento de mais estudos sobre esse e outros sistemas de microemulsão e como ele age solubilizando a parafina, é possível que ele possa vir a ser uma alternativa na remoção de depósitos parafínicos em dutos.

\section{REFERÊNCIAS}

AKBARZADEH, K.; ZOUGARI, M. Introduction to a novel approach for modeling wax deposition in fluid flows. 1. taylor-couette system. Ind. Eng. Chem. Res., v.47, p. 953-963, 2008.

AL-YAARI, M. Paraffin wax deposition: mitigation \& removal techniques. SPE 155412, SPE, 2011.

CASTRO DANTAS, T. N.; Use of Microemulsion Systems in the Solubilization of Petroleum Heavy Fractions for the Prevention of Oil Sludge Waste Formation. Energy Fuels, v. 24, p. 2312-2319, 2010.

HUANG, Z.; LU, Y.; HOFFMANN, R.; AMUNDSEN, L.; FOGLER, H. S. The effect of operating temperatures on wax deposition. Energy Fuels. v. 25, p. 5180-5188, 2011.

ROBB, I. D. Microemulsions. Plenum Press, New York, 1982.

\section{AGRADECIMENTOS}

À Universidade Federal do Amazonas (UFAM) pelo apoio. À Petrobras e ao PFRH-14 da UFRN pelo apoio financeiro e pela bolsa concedida. 